\title{
Cell Morphology Modified by Biomaterial Induces miR-21 Expression and Apoptosis
}

\author{
Mengya Liu, Shuangying Gu, Yue Zhou \\ School of Biomedical Engineering and Med-X Research Institution, Shanghai Jiao Tong University, Shanghai, China \\ Email:yzhou2009@sjtu.edu.cn
}

How to cite this paper: Liu, M.Y., Gu, S.Y. and Zhou, Y. (2016) Cell Morphology Modified by Biomaterial Induces miR-21 Expression and Apoptosis. Journal of Biosciences and Medicines, 4, 42-48. http://dx.doi.org/10.4236/jbm.2016.412007

Received: October 25, 2016 Accepted: November 24, 2016

Published: December 1, 2016

\begin{abstract}
Biochemical factors can play an important role in regulating gene expression in human umbilical vein endothelial cells (HUVECs), yet the role of biophysical factors during this process is unknown. Here, we show that physical cues, in the form of parallel microgrooves on the surface of cell adhesive substrates, can change the morphology of HUVECs as well as specific microRNA expression. Cells cultured on microgrooved poly (dimethyl siloxane) (PDMS) surface exhibited a more elongated morphology relative to those cultured on flat surfaces, and favored outgrowth along the axis of groove alignment. The level of microRNAs in the cell was screened by miRNA microchip and verified by qRT-PCR. The result showed that around $26 \mathrm{mi}-$ croRNAs have been modified significantly, among which miR-21 level was dramatically elevated. Western-blotting analysis demonstrated that PTEN, a target of miR21, was up-regulated in HUVECs with elongated morphology. Cell apoptosis level was significantly decreased, with was associated with the increasing of miR-21 level. These results suggested that biophysical factors can directly modify HUVECs morphology, thus induce miR-21 expression in HUVECs and its downstream biological functions such as decreasing apoptosis. This study provided evidence that surface microtopology should also be considered in designing biomaterials in tissue engineering application.
\end{abstract}

\section{Keywords}

HUVECs, Microgroove Topography, microRNA, Apoptosis

\section{Introduction}

In tissue engineering material research field, biochemical modification is the most popularly applied approach to enhance the material in tissue repair and regeneration [1]-[3]. However, they usually cause ethical problems and unfavorable side-effects. Stu- 
dies have shown that physical factors were also capable of regulating cellular behavior. Mechanical forces play an important role in regulating vascular signaling and gene expression in endothelial cells (ECs) to modulated cell morphology, migration, growth, proliferation, apoptosis and so on [4]. For example, blood sheer stress could modulate apoptosis in HUVECs by overexpression of miR-21 [5]. In addition, study from Downing et al. demonstrated that cell morphology change by substrate microtopology could enhance cell reprogramming by histone acetylation and methylation [6].

Since post-transcriptional regulation of genes expression was involved in most cellular processes. Expression level of miR-10a was reported to be downregulated in atherosusceptible regions of the aorta and related to inflammation [7] [8]. Some articles illustrated that miR-19a could be rapidly induced by shear stress [9] and miR-146a, miR-155 were related to inflammation [10] [11]. Considering cell migration, there are several articles reported about miR-29 [12]-[14] and miR-150 [15].

To elucidate the role of biophysical factors in regulating endothelial functions, we used the culture substrate with micro-grooves to physically change the shape of endothelial cell. We focused on the change of the miR-21 expression to reveal the role of microRNAs in regulating HUVECs function.

\section{Methods}

\subsection{Cell Culture}

Human umbilical vein endothelial cells (HUVECs) were purchased from Sciencell. The cells were cultured in ECM (Sciencell) consisted of 5\% fetal bovine serum (FBS), 1\% penicillin/streptomycin, and $1 \%$ endothelial growth factors provided by the manufacturer. Cell at passage 4 to 8 , were seeded on the PDMS membrane for 3 days before proceeding to the following experiments.

\subsection{Lithographic Silica Wafer and PDMS Membrane}

To create patterned membranes with parallel microgrooves $(10 \mu \mathrm{m}$ in width and $3 \mu \mathrm{m}$ in depth, with $10 \mu \mathrm{m}$ distance between each groove), we used microfabrication techniques [16]. Poly (dimethyl siloxane) (PDMS) was prepared according to the manufacturer protocol (Sylgard 184, Dow Corning, Midland, MI), spin-coated onto the patterned silicon wafers to desired thickness $(\sim 250 \mu \mathrm{m})$, degassed under vacuum, and cured at $70^{\circ} \mathrm{C}$ for $2 \mathrm{~h} \mathrm{[17].}$

The resulting micropatterned membranes were removed from the template, cut to appropriate dimensions and thoroughly washed and sonicated before use. The surface topography of micropatterned PDMS membranes was examined by scanning electron microscopy (SEM) [18].

\subsection{Immunohistostaining}

Antibody against $\beta$-catenin and phalloidin-fluorescein conjugate was obtained from Abcam and AAT Bioquest, respectively. DAPI was obtained from Beyotime Biotechnology. The immunohistostaining of HUVECs was examined by Confocal Laser Scan- 
ning Microscope TCS SP5II (Leica).

\subsection{Total RNA and microRNA Isolation}

We isolated total RNA and microRNA using TRIzol plus RNA Purification (Invitrogen) and miRcute miRNA isolation kit (TIANGEN) respectively following the manufacture's instruction.

\section{5. microRNA Microchip and qRT-PCR Analysis}

PureLink $^{\oplus}$ miRNA Isolation Kit (Ambion) was used to purify microRNA which then collected to run miRNA microchip (Oebiotech Co. Ltd). The chip applied in this experiment was Agilent Human miRNA $\left(8^{\star} 60 \mathrm{~K}\right)$ and analyzed by Feature Extraction software (version10.7.1.1) and Genespring software (version13.1) by Agilent Technologies.

For qRT-PCR, first-stand cDNA synthesis and quantitative assessment of microRNA was measured using miRNA first-strand cDNA synthesis kit and miRNA qPCR detection kit (CYBR green) from Tiangen, respectively. RNU6-2 was used as a normalization control in all miRNA measurements. Primers used were listed as shown in Table 1.

SuperReal PreMix Plus (SYBR Green) was used to determine the mRNA level by using the GAPDH as a normalization control. The sequences of primer were shown below: GAPDH (Forward: 5' GGG AAG GTG AAG GTC GGA GT, Reverse: 5' GGG GTC ATT GAT GGC AAC A), PTEN (Forward: 5' TTT TGA AGA CCA TAA CCC AC, Reverse: 5' TAT CAT TAC ACC AGT TCG TC).

The qPCR was run on 7900HT Fast Real-Time PCR system (Applied Biosystems).

\subsection{Western Blotting Analysis}

Whole Cell Extraction kit (Chemicon) was use to collect protein from HUVECs. Protein lysates were quantified by BCA Protein assay kit (Novagen). SDS-PAGE and polyvinylidene fluoride membranes were used to analyze the protein sample. Before incubating with primary antibodies, the membranes were blocked in 5\% non-fat milk. Primary antibodies included PTEN (ab32199, Abcam), caspase-3 (sc-7148, SANTA CRUZ) and $\beta$-Actin (\#4970, Cell Signaling). Peroxidase AffiniPure Goat Anti-Rabbit IgG $(\mathrm{H}+\mathrm{L})$ (111-035-003, Jackson immunoResearch) was used as Secondary antibody.

Table 1. Sequences of microRNA primers.

\begin{tabular}{cc}
\hline Name & Sequences \\
hsa-miR-10a-5p & UACCCUGUAGAUCCGAAUUUGUG \\
hsa-miR-19a-3p & UGUGCAAAUCUAUGCAAAACUGA \\
hsa-miR-21-5p & UAGCUUAUCAGACUGAUGUUGA \\
hsa-miR-146a-5p & UGAGAACUGAAUUCCAUGGGUU \\
hsa-miR-181a-5p & AACAUUCAACGCUGUCGGUGAGU \\
\hline
\end{tabular}




\section{Results and Discussion}

\subsection{HUVEC Morphology Change Upregulates miRNA-21 Expression}

To assess whether topography might influence the expression of microRNA, HUVECs were seeded onto flat PDMS membranes (F) or those fabricated with parallel microgrooves of $10 \mu \mathrm{m}$ width and spacing $(\mathrm{G})$. The SEM images of PDMS membranes with or without $10 \mu \mathrm{m}$ micro-grooves were shown in Figure 1(A). Phalloidin staining demonstrated that microgroove substrates had a pronounced effect on HUVEC alignment. (Figure 1(B)). In general, cells cultured on microgrooves exhibited a more elongated morphology relative to those cultured on flat surfaces, and the stress fibers was also parallel to the axis of groove alignment. The nuclear elongation was also observed.

In addition, we applied microRNA chips to screen the expression levels of we measured the expression level of a number of miRNAs, however, we observed that microgroove topography significantly increased the expression of these miRNAs (Figure 2(B)). Among these microRNAs, miR-21 was increased significantly after culturing on microgroove topography.

\section{2. miR-21 Overexpression Modulates Apoptosis in HUVECS}

According to related articles, miR-21 was demonstrated to involve in modulating the decrease of apoptosis in different adult tissues, such as HUVECs. We hypothesized that the increase of miR-21 expression caused by grooved materials could lead to the decrease of apoptosis. To test this hypothesis, HUVECs were cultured on grooved and flat PDMS; and then the protein was collected to test the expression of Procaspase- 3 and cleaved caspase-3. The activation of caspase- 3 was tested to confirm the apoptosis of HUVECs. Caspase family is important in the process of apoptosis, especially Caspase- 3 . When apoptosis happens in cells, caspase- 3 will active and divide into two small sub-units (12KD and 17KD). Procaspase-3 expression levels increased and cleaved caspase-3 (17KD) expression levels decreased in HUVECs cultured on grooved PDMS in

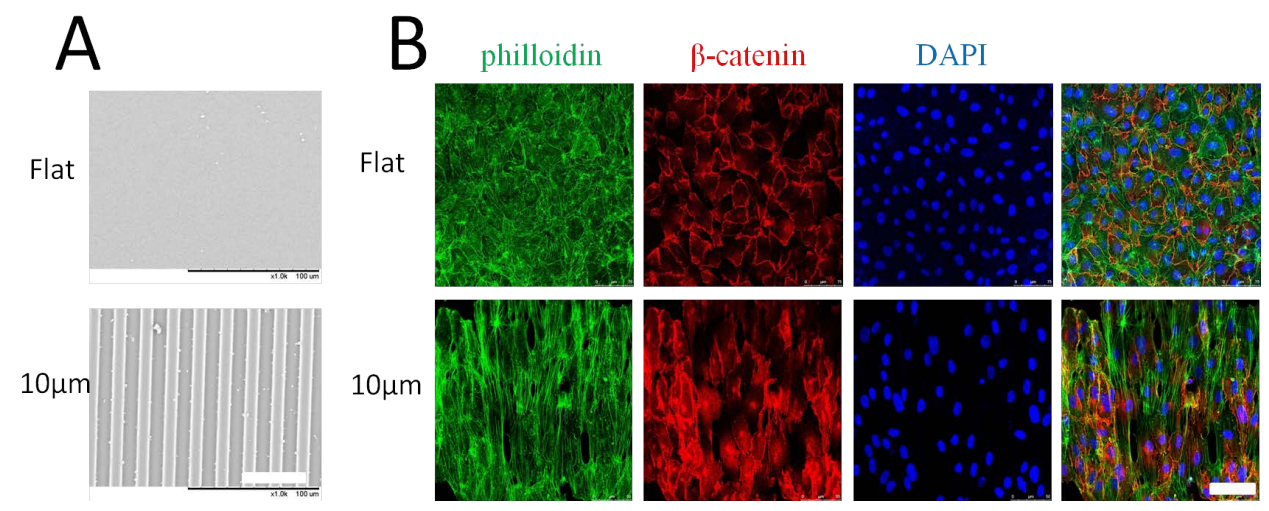

Figure 1. The phenotypes of the HUVECs were changed on PDMS membrane with microgrooved surface. (A) Scanning electron micrograph (SEM) of PDMS membranes with flat surface and grooved surface with $10 \mu \mathrm{min}$ width and $3 \mu \mathrm{m}$ in depth; (B) HUVECs cultured on flat PDMS membrane or micro-grooved surface, stained by phailloidin (green), $\beta$-catenin (red) and DAPI (blue). (Scale bar, $50 \mu \mathrm{m}$ ). 
comparison with flat PDMS (Figure 3). However the expressions of cleaved caspase-3 which is $12 \mathrm{KD}$ have no difference.

\subsection{HUVECS Change of HUVEC Morphology and miR-21 Overexpression Attenuate PTEN Expression}

Some articles showed that miR-21 was involved in expression of the proapoptotic gene PTEN. MiR-21 was illustrated to decrease the apoptosis of HUVECs and vascular smooth muscle cells by downregulation of PTEN.

Overexepression of miR-21 in HUVECs which cultured on grooved materials reduced PTEN protein expression. These results are consistent with negative regulation of PTEN by miR-21 in HUVECs (Figure 4).
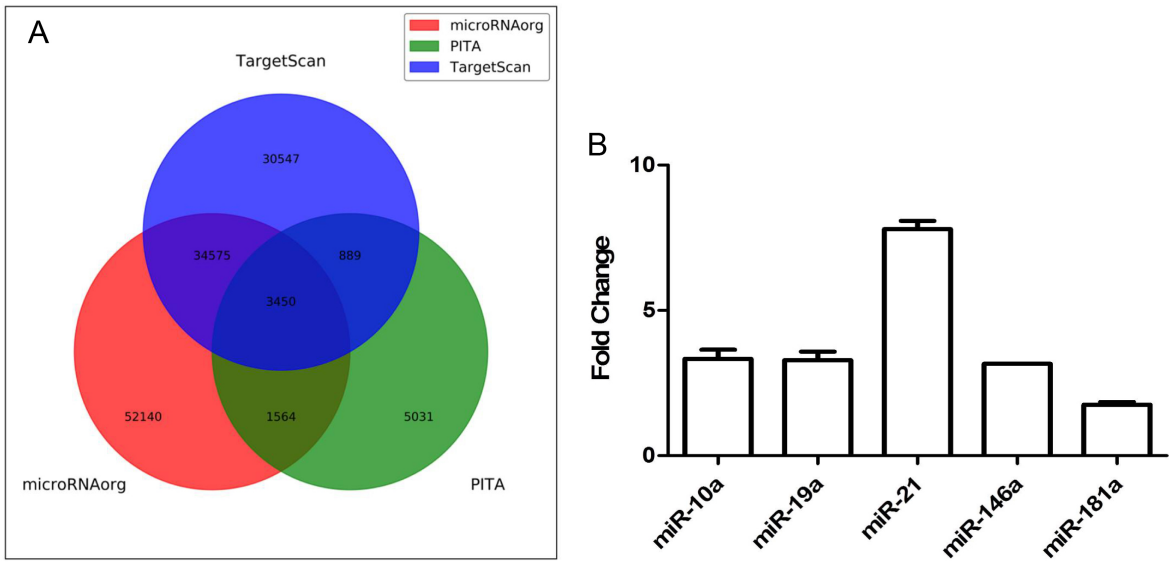

Figure 2. The microRNA expressions of the HUVECs were changed on PDMS membrane with micro-grooved surface. (A) microRNA microchip analysis results summary of HUVECs cultured on micro-grooved surface; (B) representative real-time PCR confirmation analysis of microRNA levels in HUVECs cultured on micro-grooved surface vs. flat surface control.

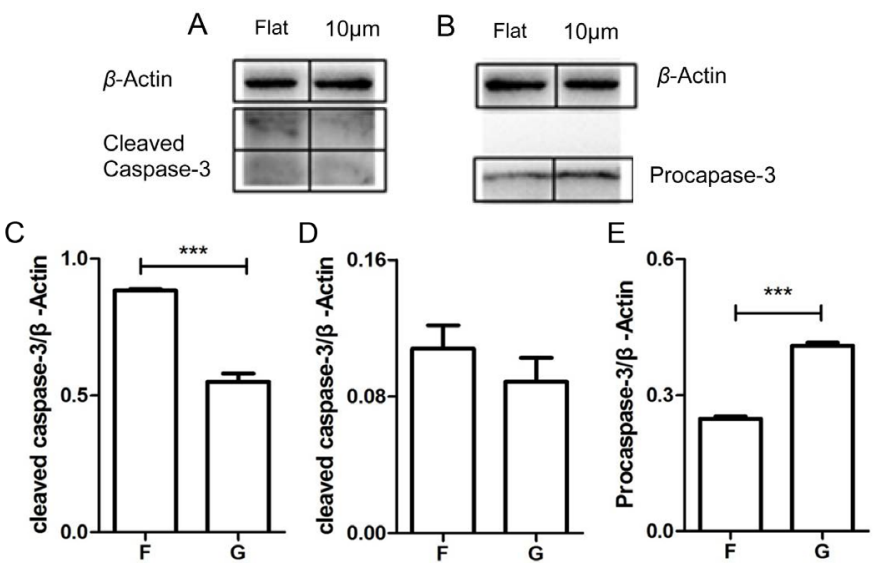

Figure 3. The procaspase-3 and cleaved caspase-3 expression in HUVECs which were cultured on PDMS with micro-grooved or flat surface. (A)-(D) Western analysis of cleaved caspase-3 in HUVECs cultured on grooved and flat materials. (B), (E) Western analysis of procaspase-3 in HUVECs cultured on grooved and flat materials. F means flat situation, G means grooved situation. 
A

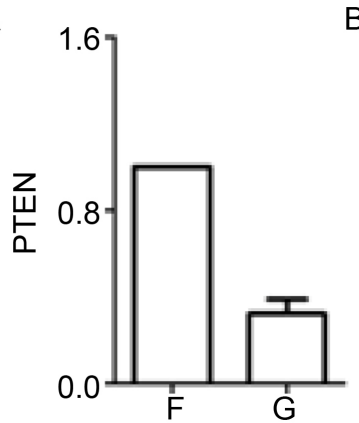

B

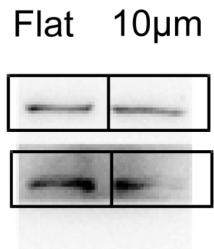

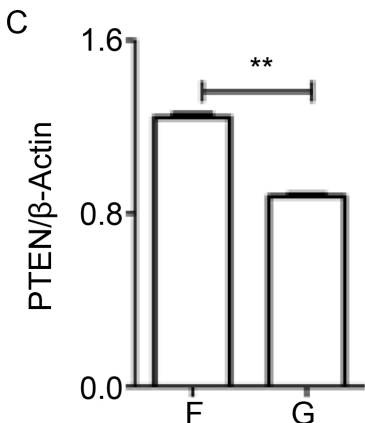

Figure 4. The PTEN expression in HUVECs which were cultured on PDMS with micro-grooved or flat surface. (A) Grooved surface significantly downregulated PTEN in HUVECs. (B), (C) Western analysis of PTEN in HUVECs cultured on grooved and flat materials. F means flat situation, $G$ means grooved situation.

\section{Conclusions}

1) The morphology of HUVECs can be changed by fine-tuning the surface topography of the material they attach.

2) HUVECs could sense the subtle changes in its morphology and response in multiple ways including cytoskeleton related proteins and microRNA expression.

3) In tissue engineering, biophysical factors are also possible to affect the apoptosis of the cells.

\section{Acknowledgements}

This research was funded by Science and Technology commission of Shanghai Municipality (16140901900).

\section{References}

[1] Akkol, E.K., Süntar, I., Orhan, I.E., et al. (2011) Assessment of Dermal Wound Healing and In Vitro Antioxidant Properties of Avena Sativa L. Journal of Cereal Science, 53, 285-290. http://dx.doi.org/10.1016/j.jcs.2011.01.009

[2] Maalej, H., Moalla, D., Boisset, C., et al. (2014) Rhelogical, Dermal Wound Healing and In Vitro, Antioxidant Properties of Exopolysaccharide Hydrogel from Pseudomonas stutzeri, AS22. Colloids \& Surfaces B Biointerfaces, 123, 814-824. http://dx.doi.org/10.1016/j.colsurfb.2014.10.017

[3] Khaing, Z.Z., Ehsanipour, A., Hofstetter, C.P., et al. (2016) Injectable Hydrogels for Spinal Cord Repair: A Focus on Swelling and Intraspinal Pressure. Cells Tissues Organs, 202. http://dx.doi.org/10.1159/000446697

[4] Chien, S. (2007) Mechanotransduction and Endothelial Cell Homeostasis: The Wisdom of the Cell. Ajp Heart \& Circulatory Physiology, 292, 135-180.

[5] Weber, M., Baker, M.B., Moore, J.P., et al. (2010) MiR-21 Is Induced in Endothelial Cells by Shear Stress and Modulates Apoptosis and eNOS Activity. Biochemical \& Biophysical Research Communications, 393, 643-648. http://dx.doi.org/10.1016/j.bbrc.2010.02.045

[6] Downing, T.L., Soto, J., Morez, C., et al. (2013) Biophysical Regulation of Epigenetic State and Cell Reprogramming. Nature Material, 12, 1154-1162.

http://dx.doi.org/10.1038/nmat3777 
[7] Fang, Y., Shi, C., Manduchi, E., Civelek, M. and Davies, P.F. (2010) MicroRNA-10a Regulation of Proinflammatory Phenotype in Athero-Susceptible Endothelium in Vivo and in Vitro. Proc. Natl Acad. Sci. USA, 107, 13450-13455.

http://dx.doi.org/10.1073/pnas.1002120107

[8] Fang, Y., Shi, C., Manduchi, E., et al. (2010) MicroRNA-10a Regulation of Proinflammatory Phenotype in Athero-Susceptible Endothelium in Vivo and in Vitro. Proc Natl Acad Sci USA, 107, 13450-13455. http://dx.doi.org/10.1073/pnas.1002120107

[9] Qin, X., Wang, X., Wang, Y., et al. (2010) MicroRNA-19a Mediates the Suppressive Effect of Laminar Flow on Cyclin D1 Expression in Human Umbilical Vein Endothelial Cells. Proceedings of the National Academy of Sciences of the United States of America, 107, 32403244. http://dx.doi.org/10.1073/pnas.0914882107

[10] Li, L., Chen, X.P. and Li, Y.J. (2010) MicroRNA-146a and Human Disease. Scand. J. Immunol., 71, 227-231. http://dx.doi.org/10.1111/j.1365-3083.2010.02383.X

[11] Fichtlscherer, S., De Rosa, S., Fox, H., et al. (2010) Circulating miRNAs in Patients with Coronary Artery Disease. CircRes, 107, 677-684. http://dx.doi.org/10.1161/CIRCRESAHA.109.215566

[12] Ugalde, A.P., Ramsay, A.J., Rosa, J.D.L., et al. (2011) Aging and Chronic DNA Damage Response Activate a Regulatory Pathway Involving miR-29 and p53. Embo Journal, 30, 22192232. http://dx.doi.org/10.1038/emboj.2011.124

[13] Martinez, I., Cazalla, D., Almstead, L.L., Steitz, J.A. and DiMaio, D. (2011) miR-29 and miR-30 Regulate B-Myb Expression during Cellular Senescence. Proceedings of the National Academy of Sciences, 108, 522-527. http://dx.doi.org/10.1073/pnas.1017346108

[14] Chen, K.C., Wang, Y.S., Hu, C.Y., et al. (2011) OxLDL Up-Regulates microRNA-29b, Leading to Epigenetic Modifications of MMP-2/MMP-9 Genes: A Novel Mechanism for Cardiovascular Diseases. Faseb Journal Official Publication of the Federation of American Societies for Experimental Biology, 25, 1718-1728. http://dx.doi.org/10.1096/fj.10-174904

[15] Zhang, Y., Liu, D., Chen, X., Li, J., Li, L., Bian, Z., et al. (2010) Secreted Monocytic miR-150 Enhances Targeted Endothelial Cell Migration. Mol Cell, 39, 133-144.

http://dx.doi.org/10.1016/j.molcel.2010.06.010

[16] Kurpinski, K. and Li, S. (2006) Anisotropic Mechanosensing by Mesenchymal Stem Cells. Proceedings of the National Academy of Sciences of the United States of America, 103, 16095-16100. http://dx.doi.org/10.1073/pnas.0604182103

[17] Downing, T.L., Soto, J., Morez, C., et al. (2013) Biophysical Regulation of Epigenetic State and Cell Reprogramming. Nature Material, 12, 1154-1162. http://dx.doi.org/10.1038/nmat3777

[18] Thakar, R.G., Ho, F., Huang, N.F., et al. (2003) Regulation of Vascular Smooth Muscle Cells by Micropatterning. Biochemical \& Biophysical Research Communications, 307, 883-890. http://dx.doi.org/10.1016/S0006-291X(03)01285-3 
Submit or recommend next manuscript to SCIRP and we will provide best service for you:

Accepting pre-submission inquiries through Email, Facebook, LinkedIn, Twitter, etc. A wide selection of journals (inclusive of 9 subjects, more than 200 journals)

Providing 24-hour high-quality service

User-friendly online submission system

Fair and swift peer-review system

Efficient typesetting and proofreading procedure

Display of the result of downloads and visits, as well as the number of cited articles

Maximum dissemination of your research work

Submit your manuscript at: http://papersubmission.scirp.org/

Or contact jbm@scirp.org 